\title{
BMJ Open Discussing prognosis and end-of-life care in the final year of life: a randomised controlled trial of a nurse-led communication support programme for patients and caregivers
}

\author{
Adam Walczak, ${ }^{1}$ Phyllis N Butow, ${ }^{1}$ Josephine M Clayton, ${ }^{1,2}$ Martin H N Tattersall, ${ }^{1}$ \\ Patricia M Davidson, ${ }^{3}$ Jane Young, ${ }^{4}$ Ronald M Epstein ${ }^{5}$
}

To cite: Walczak A, Butow PN, Clayton JM, et al. Discussing prognosis and end-of-life care in the final year of life: a randomised controlled trial of a nurse-led communication support programme for patients and caregivers. BMJ Open 2014;4: e005745. doi:10.1136/ bmjopen-2014-005745

- Prepublication history for this paper is available online. To view these files please visit the journal online (http://dx.doi.org/10.1136/ bmjopen-2014-005745).

Received 21 May 2014 Accepted 6 June 2014

CrossMark

For numbered affiliations see end of article.

Correspondence to Adam Walczak; adam. walczak@sydney.edu.au

\section{ABSTRACT}

Introduction: Timely communication about life expectancy and end-of-life care is crucial for ensuring good patient quality-of-life at the end of life and a good quality of death. This article describes the protocol for a multisite randomised controlled trial of a nurse-led communication support programme to facilitate patients' and caregivers' efforts to communicate about these issues with their healthcare team.

Methods and analysis: This NHMRC-sponsored trial is being conducted at medical oncology clinics located at/affiliated with major teaching hospitals in Sydney, Australia. Patients with advanced, incurable cancer and life expectancy of less than 12 months will participate together with their primary informal caregiver where possible. Guided by the self-determination theory of health-behaviour change, the communication support programme pairs a purpose-designed Question Prompt List (QPL—an evidence-based list of questions patients/caregivers can ask clinicians) with nurse-led exploration of QPL content, communication challenges, patient values and concerns and the value of early discussion of end-of-life issues. Oncologists are also cued to endorse patient and caregiver question asking and use of the QPL. Behavioural and self-report data will be collected from patients/caregivers approximately quarterly for up to 2.5 years or until patient death, after which patient medical records will be examined. Analyses will examine the impact of the intervention on patients' and caregivers' participation in medical consultations, their self-efficacy in medical encounters, quality-of-life, end-of-life care receipt and quality-ofdeath indicators.

Ethics and dissemination: Approvals have been granted by the human ethics review committee of Royal Prince Alfred Hospital and governance officers at each participating site. Results will be reported in peer-reviewed publications and conference presentations.

Trial registration number: Australian New Zealand Clinical Trials Registry ACTRN12610000724077.

\section{Strengths and limitations of this study}

- Encourages early discussion of prognosis and end-of-life care.

- Novel combination of previously successful interventions.

- Addresses all facets of self-determination theory of health behaviour change.

- Targets multiple stakeholders.

- Rigorous study design utilising objective and self-report data.

- Challenging to enforce endorsement of question asking and Question Prompt List.

- Doctors do not receive comprehensive training in responding to patient and caregiver questions.

- Intervention effect may not be fully captured in single recorded consultation.

\section{INTRODUCTION}

Despite treatment advances, cancer often remains a lethal disease. Disease progression, patterns of decline and death are somewhat predictable. Knowing one's life expectancy and planning care may afford opportunities to prepare for the end of life (EOL) and ensure care is consistent with patients' values. Thus timely doctor/patient communication about prognosis and EOL issues is vital.

Advance Care Planning (ACP) can guide such communication. This process involves discussion between a patient, their family and clinicians to clarify and reflect on values, treatment preferences and goals to develop a shared understanding of how EOL care should proceed. ${ }^{1}$ Discussing the patient's prognosis often forms a component of ACP. This can clarify the timing and progression of the patient's decline and focus communication on how to balance goals of care. Such 
discussions may reduce aggressive EOL medical care and associated costs, ${ }^{2}$ increase early hospice referral, ${ }^{3}$ increase patient and caregiver satisfaction with care and improve quality of life (QOL) and survival. ${ }^{45}$

This study is a randomised controlled trial (RCT) of a nurse-led communication support programme (CSP) for patients with advanced cancer and their primary informal caregiver. It is structured around a purposedesigned Question Prompt List (QPL) highlighting the value of early discussion of prognosis and EOL care. The intervention seeks to improve communication between patients, caregivers and oncologists regarding these issues and open dialogue regarding ACP. This article describes the rationale, evidence base and theoretical foundations and structure of the CSP, together with details of study outcomes, hypotheses, measures and administration protocols. Planned analyses, ethical considerations and dissemination will also be discussed.

\section{Communication about prognosis and EOL issues}

Communicating about prognosis and EOL issues can be challenging for doctors and patients. Doctors' avoidance of life-expectancy discussions is common, ${ }^{6}$ often for fear of destroying hope or the therapeutic relationship. ${ }^{7}$ Patients' readiness to discuss such issues is variable, and depends on factors including degree and length of exposure to their disease, having time to adjust to the cancer diagnosis, coping style and spirituality. ${ }^{8}$ Death anxiety may also trigger defence mechanisms such as denial or avoidance to minimise anxiety and distance the mortality threat. ${ }^{9-13}$ Consequently, doctors and patients may 'collude' to avoid these discussions. ${ }^{14}$ Communication about EOL care often first occurs near death during acute hospital admissions, ${ }^{15}$ when critical decisions about chemotherapy and aggressive life support may have been made. Research suggests as few as $14 \%$ of doctors know patients' preferences regarding pain management or place of death, ${ }^{16}$ and many patients receiving palliative chemotherapy do not understand its non-curative intent. ${ }^{17}$ Hence, such discussions can be too late or of poor quality.

Evidence suggests that EOL discussions do not increase anxiety ${ }^{18} 19$ and a lack of information may heighten anxiety by raising patients' suspicions that doctors are withholding potentially frightening information. ${ }^{19}{ }^{20}$ While the majority of patients with incurable cancer indicate they want prognostic and EOL care discussions, ${ }^{21} 22$ prognostic information is not universally desired. Eliciting preferences can be complex and may impact subsequent EOL care discussions although patients' EOL priorities often relate to effective communication. One study found that patients' highest ranked attributes of a good death included to 'have a nurse with whom one feels comfortable', 'know what to expect about one's physical condition', 'have someone who will listen', 'trust one's physician', 'have physician with whom one can discuss fears', 'know that one's physician is comfortable talking about death and dying' and 'feel prepared to die'.23

\section{Self-determination theory}

An ethical imperative to open prognostic disclosure and early EOL discussions is the principle of patient autonomy; that patients have the right to information about their body and to contribute to decisions about their medical care if they wish. ${ }^{24}$ The self-determination theory of health-related behaviour change ${ }^{2526}$ suggests that autonomy is not only a basic human right, but that meeting patients' needs for autonomy, as well as for competence and relatedness, enhances engagement in care and maintenance of outcomes. Thus it is critical to address patient and health professional barriers to EOL communication, and to involve patients in tailoring communication to best suit their needs.

\section{Caregiver involvement}

Family caregivers play a crucial role in the EOL care of patients with advanced disease. Beyond providing physical support, caregivers may act as medical decision surrogates in a formal or informal capacity. Caregivers often do not receive sufficient information regarding patient disease status and prognosis from clinicians or discuss EOL care preferences with patients and adequately prepare for surrogate decision-making. ${ }^{27}$ This may hinder their ability to make appropriate decisions regarding patient care. Patients and caregivers also have somewhat divergent information needs as the patient's illness progresses, with caregivers preferring more information and patients less. ${ }^{27}$ Appropriate EOL communication has also been associated with better caregiver bereavement outcomes, less aggressive patient treatment patterns and better QOL. ${ }^{3}$

\section{Interventions to improve communication at the EOL}

Evaluations of interventions to improve communication with patients with advanced cancer have focused on impacting patient or doctor behaviours separately or the shared health communication environment. Few have targeted or involved caregivers or family. Patient interventions have generally focused on providing tools, such as QPLs, or training to increase assertiveness in meeting communication needs. QPLs are evidence-based lists of questions grouped into themes, which patients and/or caregivers are encouraged to ask their clinician. QPLs are often valued for showing the range of topics for discussion, assisting with question formulation and highlighting clinicians' willingness to discuss all subjects. ${ }^{28-30}$

Our group evaluated the impact of giving patients a QPL in the first, second or third palliative care consultation in an RCT. ${ }^{29}$ Compared with controls, patients and caregivers who received a QPL asked twice as many questions, particularly about prognosis and EOL issues, provided the doctor endorsed question asking. Intervention patients' question asking increased by 1.6 times with any endorsement of question asking from the doctor, and by 5.8 times if there was more extensive endorsement. Fewer intervention patients had unmet information needs about the future, the area of greatest unmet information need. No intervention effect was observed for 
satisfaction with care or anxiety and outcomes such as patient QOL, actual care and caregiver outcomes were not assessed.

In another study, patients with a QPL endorsed by their clinician were significantly less anxious after the consultation and recalled significantly more information about treatment issues and side effects than patients with a QPL alone. ${ }^{31}$ Notably, when physicians did not endorse the QPL, patients asked no more questions than controls highlighting the role of clinicians in determining the success of patient interventions.

Doctor interventions typically focus on communication skills training. Back $e t a \hat{l}^{2}$ reported in a pre-post study that communication skills training for oncology fellows improved skills in breaking bad news and discussing transitions to palliative care. The impact on patients of these changes was not assessed and the intervention was a 4-day residential workshop, which may not feasible for many busy clinicians.

Interventions targeting the shared health communication environment have evaluated the efficacy of a communication broker who, trusted by the oncologist and patient, can help both parties in clarifying and communicating assumptions and expectations. The seminal SUPPORT study $^{33}$ involved an RCT of a nurse-led intervention for hospitalised patients with life-limiting illnesses including advanced cancer. A trained nurse had several contacts with patients, family, physicians and hospital staff to elicit patient preferences, improve understanding of outcomes and facilitate ACP. No improvement in patient-physician communication or outcomes including incidence or timing of DNR orders, physicians' knowledge of patients' resuscitation preferences, days in intensive care unit, mechanical ventilation receipt, use of hospital resources or patients being comatose before death was seen. Specific communication tools to assist patients were not utilised and physicians were not directly targeted with communication skills training or question-asking endorsement cues, which may have reduced its impact.

In summary, external prompts normalising and placing EOL issues on the consultation agenda may be beneficial, together with efforts to improve patients' and caregivers' self-efficacy in meeting their information and involvement needs. Providing patients with cancer and caregivers with a preconsultation QPL may be helpful, combined with improving doctors' endorsement of question asking. An outside communication broker coaching patients and caregivers to clarify their questions and concerns, improving their skills in requesting information during consultations and empowering them to do so may further improve communication. Given time burdens inherent in residential training workshops for clinicians and the potential for QPLs with endorsement to improve patients' ability to get their information needs met, a doctor-endorsed QPL amplified by nurse-led communication support and information about EOL issues has promise. This powerful combination has never previously been evaluated.
Current investigation

Building on this literature, an intervention targeting patients and caregivers with the aim to improve doctorpatient-caregiver communication about prognosis and EOL issues will be evaluated. The intervention targets patients and caregivers prior to patients becoming critically ill and unable to communicate their preferences and wishes. The intervention seeks to influence the three areas identified by self-determination theory as being crucial for patients to enact and maintain ACP behaviours 252634 : (1) 'Autonomous Motivation' to discuss prognosis and EOL care preferences; (2) 'Competence' to undertake such discussions and (3) social support or 'relatedness' to participate in such discussions.

The goal of the intervention is to impact on (1) communication, (2) care, QOL and quality of death and (3) satisfaction outcomes. We hypothesise that the intervention will:

- Increase patients' and caregivers' self-reported perceived efficacy scores in communication with their oncologist (Hla);

- Increase the number of questions/cues/concerns expressed by patients and caregivers during a follow-up oncology consultation regarding the issues targeted in the QPL and overall (H1b);

- Increase concordance between (1) patients' and caregivers' self-reported preferred level of information receipt and involvement in decisions about care and (2) their self-reported actual level of these factors during a follow-up oncology consultation (H1c);

- Increase concordance between (1) patients' selfreported hopes for treatment and preferences regarding EOL medical care and (2) caregivers' self-reported understanding of patients' hopes and preferences (H1d);

- Decrease the number of high impact EOL medical interventions that patients indicate they wish to receive and increase the number of relevant others that patients will report having having communicated with regarding their preferences (H2a);

- Decrease the number of emergency room visits and high-impact medical interventions received by patients at the recruiting hospital during the last month of life (H2b);

- Increase patients' quality-of-death scores as rated by their caregiver $(\mathrm{H} 2 \mathrm{c})$;

- Increase patients' and caregivers' self-reported QOL scores $(\mathrm{H} 2 \mathrm{~d})$;

- Be rated as highly satisfactory by patients and caregivers (H3a).

\section{METHODS AND ANALYSIS}

\section{Setting and design}

This study is a parallel group RCT conducted at medical oncology clinics based at or affiliated with major hospitals in the Sydney, Australia. It will evaluate the effects of an intervention designed to improve communication 
between patients with advanced cancer, their primary informal caregivers and their oncologist. The intervention is intended for use when patients have a year or less to live.

Patients and caregivers participate as dyads. Patients can participate without a caregiver; however caregivers cannot participate without a corresponding patient participant. Patients and caregivers complete questionnaires following consent, are randomised and participate in intervention or control conditions, allow one regular oncology follow-up consultation to be audio recorded, and complete follow-up questionnaires at regular intervals until the patient's death (see figure 1). Additionally, patients' medical records are reviewed and caregivers are invited to participate in a structured interview following the patient's death.

\section{Participants}

Currently practicing medical oncologists at participating hospitals are requested to refer consecutive eligible patients to the research team after briefly mentioning the goals of the study and seeking verbal approval to release their contact details.

Patients are eligible if they have been diagnosed with any type of cancer and their medical oncologist believes they have a life expectancy of between 2 and 12 months. Caregivers are eligible if they are identified as the primary, informal providers of care to a patient participating in the trial. Patients and caregivers must read and speak English well enough to be interviewed and complete questionnaires without the aid of an interpreter, be over the age of 18 years and be capable of giving informed consent. Patients and caregivers will be excluded if they do not speak English or have significant psychological morbidity or cognitive impairment.

\section{Recruitment}

Oncologist referrers are informed about the study at departmental meetings or via a letter from the study investigators. Those who agree to refer are given all necessary information and consent forms, questionnaires and referral documents. Referrers identify patients who fit the study inclusion criteria, inform them about the study during a consultation, provide relevant study information and refer interested patients to a research nurse following the consultation. Referrers will receive regular prompts from study personnel to review their patient lists for potentially suitable participants and such opportunities will be used to discuss the ongoing challenges with referral and potential solutions. Regular study newsletters including updates on recruitment targets will also be sent to referrers to foster engagement in the study.

The research nurse explains the study in further detail and provides patients who agree to participate with information and consent forms, baseline questionnaires and a reply paid envelope to return completed study documents. If the research nurse is not present during consulting hours, the referrer sends the patient's contact details to the research team after obtaining verbal consent for the referral. A member of the research team then contacts the patient to explain the study and post the study documents to those willing to participate. The patient is requested to complete and return the forms as soon as possible by reply paid post.

Patients are told that caregivers are encouraged to participate and asked to identify the family member or friend who assists them most often with their healthcare. If a caregiver is present at the point of referral and the patient is willing for their caregiver to be involved, information about participation is provided. If no caregiver is present at the point of referral but a caregiver is nominated, a member of the research team contacts the caregiver by phone to explain the study, obtain consent and post questionnaires.

\section{Randomisation}

Patients and caregivers are randomised as dyads, with the patient as the unit of randomisation, stratified by the oncologist. Patients are randomised alone if they do not nominate a caregiver or if their nominated caregiver declines. Patients and dyads are randomised immediately following receipt of consent and baseline data.

A computer-generated random number table is used to generate 1:1 balanced randomisation code blocks for each referring oncologist. Odd numbers will indicate control arm assignment and even numbers will indicate intervention arm assignment. Each code is placed in an

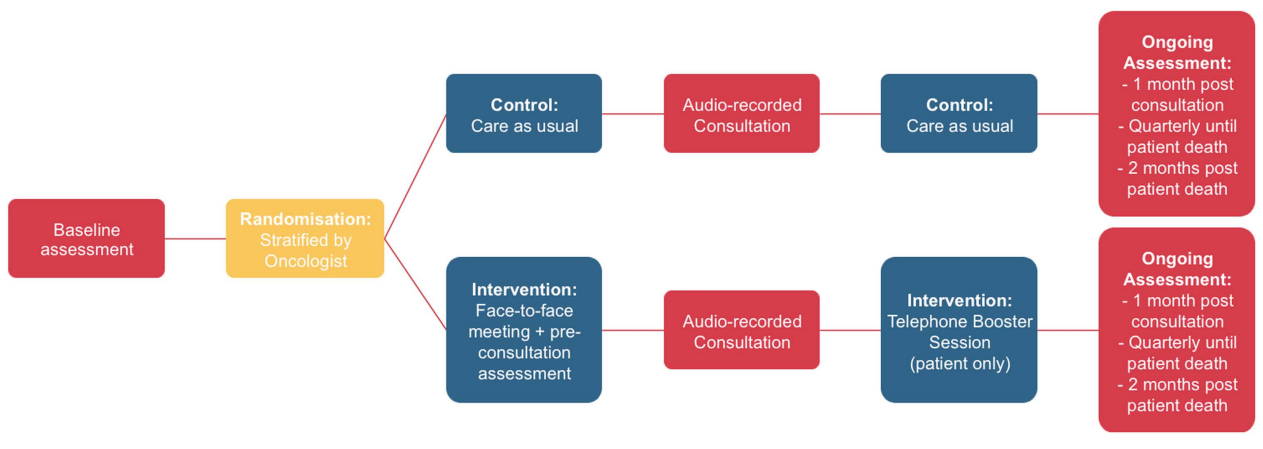

Figure 1 Study diagram. 
individual, sequentially numbered, opaque envelope. Once consent and baseline questionnaires are received, the research manager, blinded to participant identity, opens the next envelope in sequence for the referring oncologist to determine randomisation. The research staff member responsible for contacting the participant is informed of the outcome and initiates the next steps of participation.

\section{Intervention}

\section{Nurse role and training}

The intervention places primary emphasis on communication about prognosis, general EOL issues and ACP. The programme is delivered by a senior nurse who has experience in cancer care and highly developed communication skills, affording them the best opportunity to understand and relate to the experiences of patients with advanced cancer. The role of the nurse is to assist and empower patients and their caregivers to communicate with their healthcare team regarding goals, wishes, needs, concerns and questions about life expectancy and EOL issues. The nurse will not answer questions about the patient's health or individual situation but will encourage them to direct those questions to their healthcare team.

The nurse receives specific training to deliver the programme. This training involves review of a standardised training manual describing the rationale, goals and specific tasks involved in completing the intervention, followed by role-play exercises conducted with a simulated patient and directed by a researcher well versed in such training methods. Role-play exercises aim to progressively build confidence and competence in delivery and adherence to the intervention protocol. Training takes approximately $30-40 \mathrm{~h}$ per nurse, depending on the trainee's level of existing communication skills.

The study manager will regularly review recordings of sessions delivered by each trained nurse to ensure adherence to the programme delivery protocol. Any problems with adherence or programme delivery will be discussed at fortnightly meetings between the trained nurses and study manager. Nurses will also be offered the opportunity for debriefing and peer supervision with a senior psychologist as necessary.

\section{Communication Support Programme}

The CSP was informed by the self-determination theory of health-related behaviour change. In line with previous research describing ACP as a process of health behaviour change, ${ }^{34}$ discussion of prognosis, EOL care and ACP were characterised as health-related behaviours impacting EOL QOL. The intervention seeks to influence: (1) 'Autonomous Motivation' to discuss prognosis and EOL care preferences-by helping participants identify the value and importance of such discussions, (2) 'Competence' to undertake such discussions-by giving participants the skills and tools to engage in discussions and overcome barriers to planning care and (3) social support (relatedness) to participate in such discussions-by cueing oncologists to endorse question asking and use of the QPL.

Two nurses have been trained to deliver the intervention, which consists of two sessions: (1) a face-to-face meeting and (2) a telephone booster session. Face-to-face meetings include a QPL designed for patients with advanced, incurable cancer and their primary informal caregivers. ${ }^{35}$ This includes questions related to factors identified as important at EOL by patients, family, physicians and clinicians ${ }^{23}$ and suggested and developed by an expert panel. These include prognosis and disease status, treatment options and decisions, palliative care, lifestyle changes, support for the patient and their family, ACP and questions specifically for caregivers. Face-to-face meetings are delivered at cancer treatment centres and attended by patient participants, with primary informal caregiver participants also attending where possible. Prescribed face-to-face meeting content is presented in table 1 .

Telephone booster sessions aim to amplify the content of the face-to-face meeting and include review of what was discussed in the patient's last medical oncology consultation, exploration of unaddressed information needs and concerns and guidance on how to prepare for future consultations using the QPL. Booster sessions are scheduled for a time convenient to the patient between 1 and 2 weeks after the first oncology consultation following the face-to-face meeting. Caregivers are not included in this portion of the intervention. Prescribed session content is presented in table 2.

Face-to-face meetings and telephone booster sessions will be discontinued if patients or caregivers become significantly distressed at any time during the session or request that the session be stopped. Where necessary, participants will be referred to support services or counselling at participating hospitals and clinics. All such events will be recorded in an adverse events log and will be reported in accordance with the adverse event reporting procedures specified by the relevant ethics and governance committees for each site.

Oncologists are cued to endorse question asking during audio-taped follow-up consultations and to recognise the QPL and endorse participants' efforts to use it. An example of how oncologists could endorse question asking and the QPL is presented below:

I believe you met with (name of nurse) a week or so ago and received a booklet with some suggestions about helpful questions to ask. It's important that you feel you've got all the information you want. It's not unusual to leave an appointment and realise that you've forgotten to ask about something important and I want you to remember that I'm happy to answer any questions you have as best as I can. They could be questions from the booklet or something else that you've thought of or even something we covered today. And don't worry if it's too big to get through today; we can make sure we discuss it 
Table 1 Communication support programme content-sections and specific goals of the face-to-face meeting

\begin{tabular}{|c|c|c|}
\hline & Section & Specific goals \\
\hline 1 & $\begin{array}{l}\text { Introduction and discussing the meeting } \\
\text { agenda }\end{array}$ & $\begin{array}{l}\text { Explain the reason and goals of the session } \\
\text { Outline what will be discussed during session } \\
\text { Highlight procedural matters (eg, recording of the session) } \\
\text { Offer to answer questions }\end{array}$ \\
\hline 2 & $\begin{array}{l}\text { Getting to know the patient and their } \\
\text { caregiver }\end{array}$ & $\begin{array}{l}\text { Discuss patients' and caregivers' cancer journey } \\
\text { Discuss impact of cancer on life } \\
\text { - Discuss goals, wishes and concerns in light of cancer }\end{array}$ \\
\hline 3 & Introducing the question prompt list & $\begin{array}{l}\text { Give participants the QPL } \\
\text { Explain the purpose and how to use QPL } \\
\text { Highlight prognosis and end-of-life care planning content }\end{array}$ \\
\hline 4 & $\begin{array}{l}\text { Exploring the question prompt list and } \\
\text { healthcare communication }\end{array}$ & $\begin{array}{l}\text { Discuss QPL content in detail } \\
\text { Explore questions, concerns and barriers to using QPL } \\
\text { Explore experiences of communicating with the oncologist } \\
\text { Provide additional information and discussion about ACP (DVD and } \\
\text { Information sheet) and Enduring Guardianship (Australian Guardianship } \\
\text { Tribunal material) }\end{array}$ \\
\hline 5 & $\begin{array}{l}\text { Wrapping up and nominating questions } \\
\text { for the next consultation }\end{array}$ & $\begin{array}{l}\text { Assist patient to plan for the next consultation by choosing questions they } \\
\text { may like to ask and discussing how and when to ask questions } \\
\text { Offer to answer questions }\end{array}$ \\
\hline
\end{tabular}

further at a future appointment. So, do you have any questions you'd like to ask?

\section{Data collection}

\section{Assessment timing and behavioural data collection}

Intervention and control participants are randomised after completing baseline questionnaires. Intervention arm participants receive the CSP 1-2 weeks before a regular oncology follow-up consultation. Intervention arm participants complete measures at the conclusion of the face-to-face session. Following the intervention, or baseline assessment for control patients and dyads, participants' next oncology consultation is audio taped for transcription and analysis. Participants complete questionnaires approximately 1 month after the consultation is audio taped, and every 3 months until the patient's death. Two months after the patient dies, the caregiver is invited to complete a telephone interview and patients' medical records are accessed for review (see figure 1).

\section{Patient questionnaire measures}

Table 3 lists all study outcomes, questionnaires and timing of administration for patient participants. Demographic details collected from patients include age, gender, education and work details, primary diagnosis, disease-directed treatments received, medical or allied health training, ethnicity and religious affiliations. Communication self-efficacy is measured with the Perceived Efficacy in Patient Physician Interactions (PEPPI) scale. ${ }^{36}$ $Q O L$ is measured using the Functional assessment of cancer therapy-general questionnaire (FACT-G) ${ }^{37}$ and the McGill QOL scales. ${ }^{38}$ Preferences for information and involvement in decisions about care are measured using the Degner Control Preference Scale ${ }^{39}$ and two items from the Cassileth Information Styles Questionnaire. ${ }^{40}$ Achievement of preferences for information and involvement in decisions about care are measured using modified versions of these scales worded to reflect achievement of decision and information preferences. Hopes for treatment are assessed using a purpose-designed free-response question

Table 2 Communication support programme content-sections and specific goals of the telephone booster session

\begin{tabular}{lll}
\hline Section & Specific goals \\
\hline 1 Review of last oncology consultation & Explore what questions were asked in consultation \\
& Explore what information was received in consultation \\
& Explore success or otherwise of communication strategies developed in \\
& Explore new or outstanding information & Explore unaddressed questions and concerns \\
needs and concerns & Explore new questions and concerns \\
Planning for future consultations & Assist patient in planning for next consultation by choosing questions they \\
& may like to ask and discussing how and when to ask questions \\
& Reiterate how to use QPL and how to plan for future consultations \\
& Offer to answer questions
\end{tabular}


Table 3 Patient questionnaire measures and administration timing

\begin{tabular}{|c|c|c|c|c|c|}
\hline Outcome & Measure & Baseline & Preconsultation & Postconsultation & $\begin{array}{l}\text { Quarterly } \\
\text { follow-up }\end{array}$ \\
\hline Demographics & Purpose designed & $\mathrm{X}$ & & & \\
\hline Medical communication self-efficacy & PEPPI & $x$ & $\mathrm{X}^{*}$ & $x$ & $x$ \\
\hline Quality-of-life & FACT-G, MQOL & $x$ & & $x$ & $x$ \\
\hline Topics discussed in consultations & $\begin{array}{l}\text { Purpose } \\
\text { Designed }\end{array}$ & $\mathrm{X}$ & & $\mathrm{X}$ & $\mathrm{X}$ \\
\hline Information and involvement & DCPS, CISQ & $x$ & & $x \dagger$ & \\
\hline $\begin{array}{l}\text { Disease and prognostic } \\
\text { understanding }\end{array}$ & Purpose designed & $x$ & & $x$ & \\
\hline End-of-life treatment preferences & Purpose designed & $x$ & & $\mathrm{X}$ & $x$ \\
\hline Hopes for treatment & Purpose designed & $\mathrm{x}$ & & $\mathrm{X}$ & $\mathrm{X}$ \\
\hline Acceptance and adjustment to cancer & PEACE & $x$ & & $x$ & $\mathrm{X}$ \\
\hline Consultation experiences & Purpose designed & & & $\mathrm{x}$ & \\
\hline Satisfaction & Purpose designed & & $X^{*}$ & $X^{*}$ & \\
\hline
\end{tabular}

probing realistic hopes given the patient's current state of health. Preferences for future interventions are measured with a purpose-designed scale asking patients to indicate whether they want, do not want or are unsure about whether they want each of six common high-impact EOL medical interventions. Patients are also asked to indicate the reason for their preference and who they have discussed their preference with for each intervention. Acceptance of disease is measured using the Peace, Equanimity and Acceptance in the Cancer Experience Scale (PEACE). ${ }^{41}$ Patients' understanding of their prognosis is measured using a purpose-designed questionnaire asking patients to indicate the location and spread of their disease as they understand it and to indicate what treatments may still be beneficial to them. They are also asked to give a free response indicating what their doctor has told them about their life expectancy and indicate on a 12-point Likert scale the percentage chance (in $10 \%$ increments) that they will live one and five further years, with options to refuse to answer or indicate that they are unsure. Lastly, patients are asked to rate their doctor's communication skills and manner during consultations using a purpose-designed scale. Following each patient's death, their medical records will be accessed and purposedesigned data extraction will be performed to examine evidence of active, cancer-focused treatment, emergency intervention and palliative management received during the final month of life (see table 4).

\section{Caregiver questionnaire measures}

Table 5 lists all study outcomes, questionnaires and timing of administration for caregiver participants. Demographic details collected from caregivers include relationship to the patient participant, age, gender, education, work details, receipt of caregivers' benefits, medical or allied health training, ethnicity and religious affiliation. Communication self-efficacy is measured with an adapted version of the PEPPI scale, ${ }^{36}$ reflecting the caregiver/physician relationship rather than the patient/ physician relationship. QOL is measured using the SF-36 V.2. ${ }^{42}$ Preferences for information and involvement in decisions about patient care are measured using an adapted Degner Control Preference Scale ${ }^{39}$ and two items from the Cassileth Information Styles Questionnaire, ${ }^{40}$ reflecting the caregiver's role in making decisions about patient care. Achievement of preferences for information and involvement in decisions about care is measured using modified versions of these scales worded to reflect achievement of decision and information preferences. Understanding of patients' hopes for treatment is assessed using a freeresponse question probing caregivers' understanding of the patient's realistic hopes given their current state of health. Understanding of patients' preferences for future interventions is measured with a purpose-designed scale, asking caregivers to indicate whether they believe that the patient wants, does not want or if they are unsure if the patient wants each of six common high-impact EOL medical interventions. Caregivers are also asked to indicate their beliefs about the patient's reasons for their

Table 4 Postmortem assessments

\begin{tabular}{|c|c|c|c|}
\hline Outcome & Measure & Timing & $\begin{array}{l}\text { Completed } \\
\text { by }\end{array}$ \\
\hline $\begin{array}{l}\text { Medical care } \\
\text { in the final } \\
\text { month of life }\end{array}$ & $\begin{array}{l}\text { Purpose } \\
\text { designed }\end{array}$ & $\begin{array}{l}\text { More than } \\
8 \text { weeks } \\
\text { postmortem }\end{array}$ & $\begin{array}{l}\text { Medical } \\
\text { records } \\
\text { review }\end{array}$ \\
\hline $\begin{array}{l}\text { Quality of } \\
\text { death }\end{array}$ & QODD & $\begin{array}{l}6-8 \text { weeks } \\
\text { postmortem }\end{array}$ & $\begin{array}{l}\text { Caregiver } \\
\text { interview }\end{array}$ \\
\hline
\end{tabular}


Table 5 Caregiver questionnaire measures and administration timing

\begin{tabular}{|c|c|c|c|c|c|}
\hline Outcome & Measure & Baseline & Preconsultation & Postconsultation & $\begin{array}{l}\text { Quarterly } \\
\text { follow-up }\end{array}$ \\
\hline Demographics & $\begin{array}{l}\text { Purpose } \\
\text { designed }\end{array}$ & $x$ & & & \\
\hline Medical communication self-efficacy & PEPPI (adapted) & $x$ & $X^{*}$ & $x$ & $x$ \\
\hline Quality of life & SF-36 V2 & $x$ & & $\mathrm{X}$ & $x$ \\
\hline Information and involvement & $\begin{array}{l}\text { DCPS, CISQ } \\
\text { (adapted) }\end{array}$ & $\mathrm{X}$ & & $x \dagger$ & \\
\hline $\begin{array}{l}\text { Understanding of patient's disease and } \\
\text { prognosis }\end{array}$ & $\begin{array}{l}\text { Purpose } \\
\text { designed }\end{array}$ & $x$ & & $x$ & \\
\hline $\begin{array}{l}\text { Understanding of patient's end-of-life } \\
\text { treatment preferences }\end{array}$ & $\begin{array}{l}\text { Purpose } \\
\text { designed }\end{array}$ & $x$ & & $x$ & $x$ \\
\hline $\begin{array}{l}\text { Understanding of patient's hopes for } \\
\text { treatment }\end{array}$ & $\begin{array}{l}\text { Purpose } \\
\text { designed }\end{array}$ & $x$ & & $x$ & $x$ \\
\hline Satisfaction & $\begin{array}{l}\text { Purpose } \\
\text { designed }\end{array}$ & & $X^{*}$ & $X^{*}$ & \\
\hline
\end{tabular}

preferences and who the patient has discussed their preferences with for each intervention. Caregivers' understanding of the patients' prognosis is measured using a purpose-designed questionnaire, asking caregivers to indicate the location and spread of the patient's disease as they understand it and to indicate what treatments they believe may still be beneficial to the patient. They are also asked to give a free response indicating what the doctor has told them about the patient's life expectancy and indicate on a 12-point Likert scale the percentage chance (in $10 \%$ increments) that the patient will live one and five further years, with options to refuse to answer or indicate that they are unsure. Following the patient's death, caregivers are invited to participate in a structured telephone interview, the Quality Of Death and Dying scale (QODD) ${ }^{43}$ to elicit their views on the quality of the patient's death, and their satisfaction with care at that time (see table 4 ).

\section{Coding of audio-taped consultations}

Audio-taped follow-up oncology consultations are transcribed and coded using a purpose-designed, manualised coding scheme allowing content to be utilised in quantitative analyses. For each transcript, all patient and caregiver utterances indicating a need for information or discussion are identified and coded. Summaries of coded utterances are produced for each consultation (see table 6).

\section{Planned analyses}

The primary self-reported communication outcome is patients' medical communication self-efficacy, measured by the PEPPI. The primary behavioural communication outcome is the number of questions, cues and concerns raised by the patient and caregiver during the audio-taped follow-up oncology consultation. Secondary outcomes include all other questionnaire measures completed by patients and caregivers, details of patient medical care in the last month of life revealed by medical records review and the quality of death and dying interview completed by caregivers.

Analysis will be by intention to treat, using all available data from randomised participants based on group assignment. Initial analyses will examine whether randomisation produced comparable groups in terms of age, gender, education and disease factors. Confounding variables will be accounted for in subsequent analyses. The primary self-report communication outcome will be tested based on the difference between patients' score on the PEPPI at baseline and postintervention time points in the two study arms. The primary behavioural communication outcome will be tested based on counts of questions, cues and concerns expressed by patients and caregivers during the recorded consultation in the two study arms. Primary outcomes will be analysed for group differences using a mixed model with the oncologist as a random effect, to allow for comparison of consultations involving recipients of the CSP or control conditions by the same oncologist. Secondary self-report outcomes will be analysed using similar methods. Hopes for treatment and prognostic understanding of freeresponse questions will be categorically coded, allowing for the inclusion of variables in the secondary analyses, and analysed in depth using qualitative methods at a later date along with qualitative data from recorded consultations and intervention sessions. As missing data may likely have the potential for substantial attrition in a palliative population, ${ }^{44}$ the nature of missing data will be examined and addressed using methods such as listwise deletion, weighted GEE (WGEE) or multiple imputation as appropriate. 
Table 6 Consultation coding scheme

\begin{tabular}{ll}
\hline Coded for each information seeking utterance & Coded as \\
\hline Type & Question/cue/concern \\
New issue & New/clarification \\
Speaker & Patient/caregiver \\
Follows QPL content & Yes/no \\
QPL item & Indicate QPL item number \\
Wording identical & Yes/no \\
Wording of utterance & Note exact wording of utterance \\
Topic area & Indicate best applicable QPL-related or non-QPL-related area \\
& (10 QPL and 9 non-QPL codes available) \\
Addressed by oncologist & Yes-immediate response/yes-delayed response/no \\
Coded for overall consultation & \\
Number of questions/cues/concerns & Separately count of each utterance type for patient and caregiver \\
Total new issues raised & Separately count for patient and caregiver \\
Total clarifications & Separately count for patient and caregiver \\
Words spoken & Separately count for patient, caregiver and oncologist \\
Consultation length & Length in minutes and seconds \\
\hline QPL, Question Prompt List. & \\
\hline
\end{tabular}

\section{Sample size}

Power calculations were based on communication during the single audio-taped oncology follow-up consultation for each patient. With a sample of 14 oncologist referrers and 10 patients per referrer (140 patients total) there will be a $98 \%$ power to detect a difference of 1.5 EOL-related questions, cues or concerns during the audio-taped consultation. This calculation assumes an average of 0.5 EOL-related questions, cues or concerns per consultation in the control group and two EOL-related questions, cues or concerns in the treatment group. This sample also gives $84 \%$ power to detect an effect size of 0.5 on the patient medical communication self-efficacy scale (PEPPI).

\section{ETHICS AND DISSEMINATION Ethical review}

This study is being conducted in accordance with the National Health and Medical Research Council of Australia National Statement on Ethical Conduct in Human Research (2007). All participants will give informed consent to participate and may withdraw at any time without impacting their treatment or relationship with the hospital or their clinical team. Collected data will be de-identified and stored securely for a minimum of 7 years. Accepted adverse event monitoring procedures will be followed and appropriate referral to psychological and support services at participants' treating hospital will be made as required.

\section{Relevance and dissemination}

This study will be among the first to evaluate an intervention simultaneously targeting patients and caregivers to improve communication about prognosis and EOL issues, while cuing oncologists to endorse question asking and the QPL. The design of the CSP seeks to recognise and address the challenging nature of such discussions and the limitations of previous studies designed to improve EOL communication. Strengths include the targeting of multiple stakeholders and the focus on empowering patients and caregivers through careful preparation by a trained nurse, endorsement by their medical oncologist and provision of a specific tool designed to support them in clarifying their questions and concerns. The study will assess whether this intervention increases patients' and caregivers' self-efficacy in asserting their information needs, and whether this translates into increased expression of information needs and thus more information from their oncologist. Provision of tailored information may improve patient and caregiver understanding of prognosis, ${ }^{45}$ and result in a greater likelihood of effective and timely ACP with corresponding improvements in QOL outcomes. ${ }^{2-5}$ Ultimately, it is hoped that this intervention will result in patients receiving EOL care that is more concordant with their preferences and values.

The brevity of the intervention, together with the incorporation of multiple previously successful intervention types are strengths that may yield a substantial impact. This study has the potential to provide a mechanism for assisting patients, health professionals and caregivers in communicating about, understanding and acting on patient preferences to ensure quality EOL care. We plan to publish the main trial outcomes related to communication and satisfaction in a single paper, with additional papers exploring the impact of the intervention and any changes in communication on care, QOL and quality of death. Further in-depth analyses of the implementation of this intervention and responses to its content will also be published and findings will be presented at various national and international conferences. 
Author affiliations

${ }^{1}$ Centre for Medical Psychology and Evidence-based Decision-making (CeMPED), The University of Sydney, Sydney, New South Wales, Australia ${ }^{2}$ HammondCare Palliative and Supportive Care Service, Greenwich Hospital, Greenwich, New South Wales, Australia

${ }^{3}$ Cardiovascular and Chronic Care Centre, Curtin University of Technology, Sydney, New South Wales, Australia

${ }^{4}$ School of Public Health, University of Sydney, Sydney, New South Wales, Australia

${ }^{5}$ Department of Family Medicine, University of Rochester Medical Centre, Rochester, New York, USA

Acknowledgements The authors gratefully acknowledge the input and support of our study nurses Frances Bellemore and Andrew Metcalfe. They would also like to thank the medical oncologists, nurses and staff at the participating clinics and hospitals who give their time to support our recruitment efforts. Finally, we would like to sincerely thank the patients, caregivers and family members past, present and future who freely give their time to participate and support participation in this trial.

Contributors AW is the study coordinator and primary PhD student and has the primary responsibility of writing and coordinating development of all study materials and all study manuscripts. PNB is the principal investigator for the NHMRC Project Grant that funds this trial and developed the original study protocol together with chief investigators JMC, MHNT, PMD, JY and RME. All authors reviewed and approved this manuscript.

Funding This study is funded by a Project Grant from the National Health and Medical Research Council of Australia (NHMRC Grant No: 571346).

\section{Competing interests None.}

Ethics approval The trial protocol and all study forms and materials have received the approval of the Lead Human Ethics Review Board at Royal Prince Alfred Hospital (Protocol Number: X10-0032 and Approval Number HREC/10/ $\mathrm{RPAH} / 51$ ) and from the governance officers at each of the participating recruitment sites.

Provenance and peer review Not commissioned; peer reviewed for ethical and funding approval prior to submission.

Open Access This is an Open Access article distributed in accordance with the Creative Commons Attribution Non Commercial (CC BY-NC 4.0) license, which permits others to distribute, remix, adapt, build upon this work noncommercially, and license their derivative works on different terms, provided the original work is properly cited and the use is non-commercial. See: http:// creativecommons.org/licenses/by-nc/4.0/

\section{REFERENCES}

1. Singer PA, Martin DK, Lavery JV, et al. Reconceptualizing advance care planning from the patient's perspective. Arch Intern Med 2009;158:879-84.

2. Zhang B, Wright AA, Huskamp HA, et al. Health care costs in the last week of life: associations with end-of-life conversations. Arch Intern Med 2009;169:480-8.

3. Wright AA, Zhang B, Ray A, et al. Associations between end-of-life discussions, patient mental health, medical care near death, and caregiver bereavement adjustment. JAMA 2008;300:1665-73.

4. Heyland DK, Allan DE, Rocker G, et al. Discussing prognosis with patients and their families near the end-of-life: impact on satisfaction with end-of-life care. Open Med 2009;3:e101-10.

5. Detering KM, Hancock AD, Reade MC, et al. The impact of advance care planning on end of life care in elderly patients: randomised controlled trial. BMJ 2010;340:c1345.

6. Lamont EB, Christakis NA. Prognostic disclosure to patients with cancer near the end of life. Ann Intern Med 2001;134:1096-105.

7. Buiting HM, Rurup ML, Wijsbek $\mathrm{H}$, et al. Understanding provision of chemotherapy to patients with end stage cancer: qualitative interview study. BMJ 2011;342:d1933.

8. Walczak A, Butow PN, Davidson PM, et al. Patient perspectives regarding communication about prognosis and end-of-life issues: How can it be optimized? Patient Educ Couns 2013;90:307-14.
9. Burke BL, Martens A, Faucher EH. Two decades of terror management theory: a meta-analysis of mortality salience research. Pers Soc Psychol Rev 2010;14:155-95.

10. Yalom ID. Existential psychotherapy. New York: Basic Books, 1980.

11. de Haes $\mathrm{H}$, Koedoot $\mathrm{N}$. Patient centred decision making in palliative cancer treatment: a world of paradoxes. Patient Educ Couns 2003;50:43-9.

12. Vos MS, de Haes JC. Denial in cancer patients, an explorative review. Psychooncology 2007;16:12-25.

13. Miller SM. When is a little information a dangerous thing? Coping with stressful events by monitoring versus blunting. In: Levine $\mathrm{S}$, Ursin $\mathrm{H}$, eds. Coping and health. New York: Plenum Press, 1980:145-70.

14. The AM, Hak T, Koeter G, et al. Collusion in doctor-patient communication about imminent death: an ethnographic study. BMJ 2000;321:1376-81.

15. Mack JW, Cronin A, Taback N, et al. End-of-life care discussions among patients with advanced cancer: a cohort study. Ann Intern Med 2012;156:204-10.

16. Desharnais S, Carter RE, Hennessy W, et al. Lack of concorance between physician and patient: reports on end-of-life care discussions. J Palliat Med 2007;10:728-40.

17. Weeks JC, Catalano PJ, Cronin A, et al. Patients' expectations about effects of chemotherapy for advanced cancer. $N$ Engl J Med 2012;367:1616-25.

18. Gattellari M, Voigt KJ, Vutow $\mathrm{PN}$, et al. When the treatment goal is not cure: are cancer patients equipped to make informed decisions? J Clin Oncol 2002;20:503-13.

19. Fallowfield LJ, Jenkins VA, Beveridge HA. Truth may hurt but deceit hurts more: communication in palliative care. Palliat Med 2002;16:297-303

20. Sardell AN, Trierweiler SJ. Disclosing the cancer diagnosis: procedures that influence patient hopefulness. Cancer 1993;72:3355-65.

21. Hagerty RG, Butow PN, Ellis PM, et al. Cancer patient preferences for communication of prognosis in the metastatic setting. J Clin Oncol 2004;22:1721-30.

22. Hagerty RG, Butow PN, Ellis PM, et al. Communicating prognosis in cancer care: a systematic review of the literature. Ann Oncol 2005;16:1005-53.

23. Steinhauser KE, Christakis NA, Clipp EC, et al. Factors considered important at the end of life by patients, family, physicians, and other care providers. JAMA 2000;284:2476-82.

24. Miles SH, Koepp R, Weber EP. Advance end-of-life treatment planning: a research review. Arch Intern Med 1996;156:1062-8.

25. Ryan RM, Deci EL. Self-determination theory and the facilitation of intrinsic motivation, social development, and well-being. Am Psychol 2000;55:68-78.

26. Ryan RM, Patrick H, Deci EL, et al. Facilitating health behaviour change and its maintenance: Interventions based on self-determination theory. Eur Health Psychol 2008;10:2-5.

27. Parker SM, Clayton JM, Hancock K, et al. A systematic review of prognostic/end-of-life communication with adults in the advanced stages of a life-limiting illness: patient/caregiver preferences for the content, style, and timing of information. J Pain Symptom Manage 2007;34:81-93.

28. Clayton JM, Butow PN, Tattersall MHN, et al. Asking questions can help: development and preliminary evaluation of a question prompt list for palliative care patients. Br J Cancer 2003;89:2069-77.

29. Clayton JM, Butow PN, Tattersall MHN, et al. Randomised controlled trial of a question prompt list to help advanced cancer patients and their caregivers to ask questions about prognosis and end-of-life care. J Clin Oncol 2007;25:715-23.

30. Bruera E, Sweeney C, Willey J, et al. Breast cancer patient perception of the helpfulness of a prompt sheet versus a general information sheet during outpatient consultation: a randomized, controlled trial. J Pain Symptom Manage 2003;25:412-19.

31. Dimoska A, Tattersall MHN, Butow PN, et al. Can a "prompt list" empower cancer patients to ask relevant questions? Cancer 2008;113:225-37.

32. Back AL, Arnold RM, Baile WF, et al. Efficacy of communication skills training for giving bad news and discussing transitions to palliative care. Arch Intern Med 2007;167:453-60.

33. The SUPPORT Principal Investigators. A controlled trial to provide care for seriously ill hospitalized patients. JAMA 1995;274:1591-8

34. Fried TR, Bullock $\mathrm{K}$, lannone $\mathrm{L}$, et al. Understanding advance care planning as a process of health behaviour change. J Am Geriatr Soc 2009;57:1547-55

35. Walczak A, Mazer B, Butow PN, et al. A question prompt list for patients with advanced cancer in the final year of life: Development and cross-cultural evaluation. Palliat Med 2013;27:779-88. 
36. Maly RC, Frank JC, Marshal GN, et al. Perceived efficacy in patient-physician interactions (PEPPI): validation of an instrument in older persons. J Am Geriatr Soc 1998;46:889-94.

37. Webster K, Cella D, Yost K. The functional assessment of chronic illness therapy (FACIT) measurement system: properties, application, and interpretation. Health Qual Life Outcomes 2003;1:79.

38. Cohen SR, Mount BM, Tomas JJN, et al. Existential well-being is an important determinant of quality of life: evidence from the McGill quality of life questionnaire. Cancer 1996;77:576-86.

39. Degner LF, Sloan JA, Venkatesh P. The control preferences scale. Can J Nurse Res 1997;29:21-43.

40. Cassileth BR, Zupkis RV, Sutton-Smith $\mathrm{K}$, et al. Information and participation preferences among cancer patients. Ann Intern Med 1980;92:832-6.
41. Mack JW, Nilsson M, Balboni R, et al. Peace, equanimity, and acceptance in the cancer experience (PEACE): validation of a scale to assess acceptance and struggle with terminal illness. Cancer 2008:112:2509-17.

42. Ware JE, Kosinski M, Gandek B. SF-36 health survey: manual and interpretation quide. Lincoln, RI: Quality Metric Inc, 2003.

43. Downey L, Curtis JR, Lafferty WE, et al. The quality of death and dying (QODD) questionnaire: empirical domains and theoretical perspectives. J Pain Symptom Manage 2010;39:9-22.

44. Jordhoy MS, Kaasa S, Fayers $\mathrm{P}$, et al. Challenges in palliative care research; recruitment, attrition and compliance: experience from a randomised controlled trial. Palliat Med 1999;13:299-310.

45. Butow PN, Dowsett S, Hagerty R, et al. Communicating prognosis to patients with metastatic disease: what do they really want to know? Support Cancer Care 2002;10:161-8. 